\title{
CIRCULAR AND LINEAR POLARIZATION INVERSE SAR IMAGING OF SIMPLE DETERMINISTIC TARGETS AND STATISTICALLY KNOWN RANDOMLY ROUGH SURFACES USING CONSTANT AND VARYING ILLUMINATION
}

\author{
J.W. Bredow, R.L. Porco, G. Hartman
}

Wave Scattering Research Center, Department of Electrical Engineerin

University of Texas at Arlington, UTA Box 19016

Arlington, Texas 76019-0016

\begin{abstract}
In order to study mechanisms of scattering and their relative contributions to SAR and inverse SAR images, a special measurement setup has been constructed in the University of Texas at Arlington bistatic anechoic measurement facility. This setup can be used to generate images in which: (1) the target is illuminated from a constant direction as the receiver is rotated in azimuth; and (2) the target is illuminated from various directions as the receiver is rotated in azimuth. Note that the latter relates closely to conventional and spotlight mode SAR in the case where the receiver and transmitter are collocated. The former is of considerable interest scientifically in that it allows one to map the effect of a spatially fixed set of induced currents and charges. In this presentation we will discuss the imaging system performance and compare images obtained using both approaches, for a variety of simple targets and the statistically known randomly rough surfaces reported previously [3].
\end{abstract}

Important and unique features of the special imaging setup are: (1) wide-bandwidth spiral antennas to achieve nearly constant beamwidth over frequency, and to reduce problems associated with polarization alignment; and (2) an antenna mount fastened to the pedestal with r.f. connection through a rotary joint.

\section{INTRODUCTION}

Previously we performed scattering measurements on statistically known randomly rough conducting surfaces, and processed the data to obtain both scattering coefficients and inverse SAR (ISAR) images, [Nance et al., 1988] and [Nance et al., 1990]. The scattering coefficient data has been very useful for assessing the performance of various surface scattering models, but the images have been difficult to interpret. For this reason we have embarked on a systematic investigation to study the relationships between image intensity and electromagnetic interaction (in terms of induced sources) for various conducting objects, beginning with simple ones.

In microwave diversity imaging of metallic objects a commonly used expression for retrieving images from a set of scattering measurements obtained over frequency and space has the form [Li et al, 1989]:

$\overline{\mathbf{E}}_{\mathrm{s}}(\overline{\mathbf{p}})=\int_{\text {sill }} \bar{\gamma}^{\prime}\left(\overline{\mathbf{r}}^{\prime}, \overline{\mathbf{p}}\right) \mathrm{e}^{\overline{\mathbf{p}} \cdot \overline{\mathbf{r}}^{\prime}} \mathrm{ds} \mathrm{s}^{\prime}$

where the object scattering function

$\bar{\gamma}^{\prime}\left(\overline{\mathbf{r}}^{\prime}, \overline{\mathbf{p}}\right)=\left\{\begin{array}{c}\hat{\mathbf{n}}\left(\overline{\mathbf{r}}^{\prime}\right) \times \overline{\mathbf{H}}_{\mathbf{i}}{ }^{0}-\left[\left(\hat{\mathbf{n}}\left(\overline{\mathbf{r}}^{\prime}\right) \times \overline{\mathbf{H}}_{\mathrm{i}}{ }^{0}\right) \cdot \hat{\mathbf{l}}_{\mathrm{r}}\right] \hat{\mathbf{l}}_{\mathbf{r}} \\ \text { for all } \overline{\mathbf{r}} \text { on } S_{\mathrm{ill}} \\ 0, \text { elsewhere }\end{array}\right\}$

$\overline{\mathbf{E}}_{\mathrm{s}}(\overline{\mathbf{p}})$ represents the scattered fields

$\overline{\mathbf{p}}=\mathrm{k}\left(\tilde{I}_{\mathbf{r}}-\overline{\mathbf{I}}_{\mathbf{t}}\right)$

$\mathrm{S}_{\text {ill }}$ is the illuminated surface region

$\overline{\mathbf{H}}_{\mathbf{i}}{ }^{\circ}$ is the incident magnetic field at the reference point

$\hat{\mathbf{n}}\left(\mathbf{r}^{\prime}\right)$ is the unit normal vector at $\overline{\mathbf{r}}$.

$\hat{\mathbf{l}}_{\mathrm{r}}$ is the unit vector in the direction of the receiver

$\hat{\mathbf{l}}_{\mathbf{t}}$ is the unit vector in the direction of the transmitter
Although this inversion expression is strictly valid only for a limited class of targets, it is commonly used to generate images of a wide variety of targets because of its simplicity. Note that the object function and the scattered fields are related by a Fourier Transformtype of relationship, i.e., Fourier and Fast Fourier techniques can be used to obtain one from the other.

For the above expression it should be noted that for conducting targets the $\widehat{\mathbf{n}}\left(\overline{\mathbf{r}}^{\prime}\right) \times \overline{\mathbf{H}}_{i}{ }^{\circ}$ term in the object function relates to sources on the target; this is a result of the boundary condition $\overline{\mathbf{J}}=\widehat{\mathbf{n}}\left(\overline{\mathbf{r}}^{\prime}\right) \times \overline{\mathbf{H}}_{i}{ }^{o}$. One implication of this is that as imaging measurements are performed in the usual monostatic or bistatic fashion (i.e., the target is rotated and the antennas remain stationary), the set of source currents on the target varies as scattering measurements are obtained. For the purpose of relating image intensities to sources on the object a better scheme is to maintain illumination from a constant direction as the receiver samples the scattered fields from various directions.

\section{FACILITY DEVELOPMENT AND MODIFICATIONS}

\section{Varying illumination}

The microwave anechoic measurement facility at the University of Texas at Arlington Wave Scattering Research Center was designed to facilitate bistatic measurements. An array of 27 receive antennas over a quarter-sphere section at constant radius from the pedestal and three transmit antennas permit 81 bistatic angle measurements of a stationary target. Hence, monostatic and bistatic data for images can be obtained using the facility as it was originally designed, i.e., by performing scattering measurements as the target is rotated.

\section{Constant illumination}

One significant drawback in the design of the UTA anechoic chamber is that it did not permit measurements for the case where only the receive antenna is moved. To facilitate imaging measurements with constant illumination direction, we fabricated a transmitter antenna mount which was made primarily of styrofoam components and rotates with the positioner pedestal. This allows us to maintain a constant spatial relationship between the transmit antenna and target, and scanning of the receive antenna occurs as the pedestal is rotated. To ensure phase coherency it was necessary to prevent motion in the cable that feeds the transmit antenna; this was accomplished by feeding the arm-mounted antenna through a rotary joint at the base of the pedestal.

In addition to hardware modifications, some software modifications have been necessary. The routines developed previously at UTA for obtaining images from scattering measurements [Dolaty, 1989] are restricted to the more conventional cases of bistatic and monostatic imaging. Originally, software was written with only the varying illumination situation in mind and projection of the image onto the $\mathrm{z}=0$ plane, which is perpendicular to the target pedestal. This being the case, the $\mathrm{k}$-space circles are concentric and interpolation of the scattering data onto a uniformly spaced grid (necessary for standard FFT routines) is straightforward. However, in the constant illumination case, the circles are no longer concentric and, depending on the angles used, there may be points where the circles overlap in the $x-y$ plane. To correct for this problem we redefine the $x, y$, and $z$ 
vectors such that the $\mathrm{z}$ axis is aligned with the transmitter. By projecting onto the new $z=0$ plane, we see in Figures 2 and 3 that the $\mathrm{k}$-space circles are once again concentric, this time for both the varying and constant illumination cases. Once this is done, the same software developed for varying illumination can be used to obtain images.
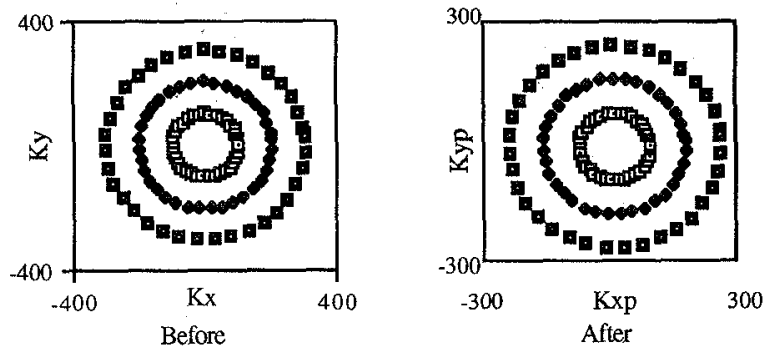

Figure 1: Varying illumination Ewald circles before and after coordinate transformation
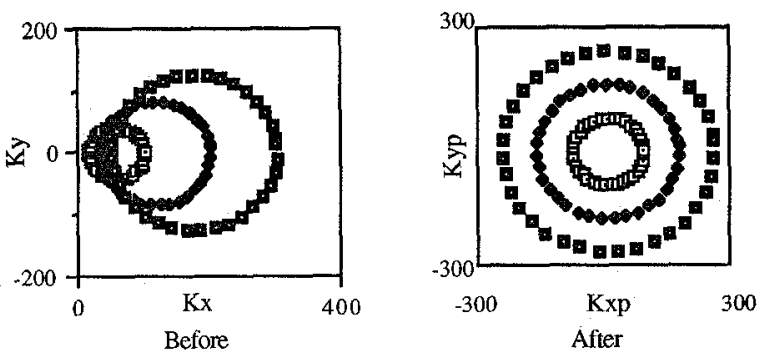

Figure 2: Constant illumination Ewald circles before and after coordinate transformation

\section{MEASUREMENTS AND DISCUSSION}

All images reported here were obtained using a frequency sweep of 4-12 GHz, elevation angles of about $50^{\circ}$, and angular measurement aperture of $90^{\circ}$. Figure 3 demonstrates the differences between the constant and varying illumination cases. Although the images are discernible in the constant illumination case, they are not as well defined as in the varying illumination situation. We have identified two possible reasons for this result: (1) there is more noise introduced in the constant illumination case due to motion of the moving antenna mount and (2) the cylinders used in the constant illumination case were larger in diameter and more closely spaced than the bistatic case. This close proximity led to more coupling between cylinders.

The circularly polarized images using varying illumination are very similar to the linear polarization images using varying illumination. This is to be expected since VV and $\mathrm{HH}$ images of the cylinder were very much alike.

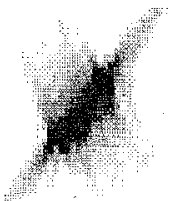

Theoretical Constant Illumination

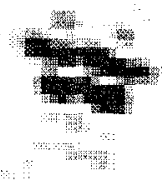

Two Cylinders (Measured) Constant Illumination

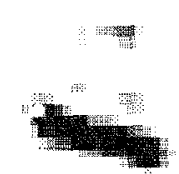

One cylinder (Measured) Constant Illumination

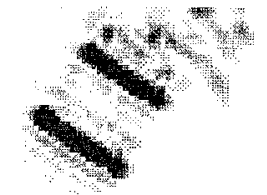

Two Cylinders (Measured) Varying Illumination

Figure 3: VV polarization

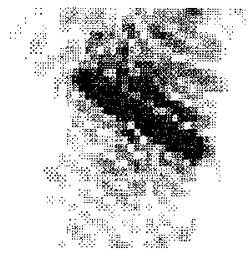

Measured

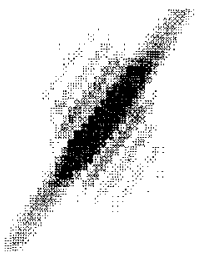

Theoretical
Figure 4: Circular polarization, varying illumination, one cylinder

\section{SUMMARY AND FURTHER WORK}

This report has considered, in a rather cursory fashion, a number of aspects pertinent to constant and varying illumination in ISAR imaging. We have considered the effects of object orientation (or angular measurement aperture), polarization, and imaging method. All of these are important effects. However, we would like to emphasize the significance of performing constant-illumination imaging measurements as a means for better understanding imaging in terms of induced sources at the target.

In this report we have indicated the theoretical basis for emphasizing constant-illumination measurements, the development of a facility for performing such measurements, and preliminary results using this facility.

In the future we expect to continue to investigate all of the problems stated above. However, we intend to make extensive comparisons between conventional bistatic or monostatic imaging and constantillumination imaging to: (1) obtain a better understanding of how image intensity relates to induced sources on the target; and (2) to better understand imaging for the conventional imaging geometries. We expect this research to improve interpretation of imaging of random targets in terms of scattering mechanisms on or within the targets.

\section{ACKNOWLEDGMENT}

This study was supported by NASA grant NAGW 2344.

\section{REFERENCES}

[1] Dolaty, M., Inverse synthetic aperture radar imaging techniques, M.S. thesis, University of Texas at Arlington, 1989.

[2] Li, H.J., N.H. Farhat, Y. Shen and C.L. Werner, "Image understanding and interpretation in microwave diversity imaging," IEEE Transactions on Antennas and Propagation, v. 37, no. 8, pp. 1048-1057, 1989.

[3] Nance, C.E., A.J. Blanchard, and M. Chen, "Polarimetric microwave scattering from known randomly rough surfaces," Proc. IGARSS '88 Symposium, Edinburgh, Scotland, 1988.

[4] Nance, C.E., A.K. Fung, and J.W. Bredow, "Comparison of integral equation predictions and experimental backscatter measurements from random conducting rough surfaces," Proc. IGARSS '90 Symposium, College Park, MD, 1990. 